\title{
Fermi-LAT studies of IceCube's track-like HESE events.
}

\author{
Anthony M. Brown * \\ Department of Physics, Durham University, DH1 3LE, UK \\ Department of Physics and Astronomy, University of Canterbury, Christchurch 8140, New \\ Zealand \\ E-mail: anthony.brown@durham.ac.uk

\section{Jenni Adams} \\ Department of Physics and Astronomy, University of Canterbury, Christchurch 8140, New \\ Zealand \\ E-mail: jenni.adams@canterbury.ac.nz
}

\section{Paula M. Chadwick}

Department of Physics, Durham University, DH1 3LE, UK

E-mail: p.m.chadwickedurham.ac.uk

\begin{abstract}
A small subset of IceCube's extraterrestrial neutrino candidates are detected as track events. The track-like nature of these events within the IceCube detector affords us a $\sim 1$ degree angular resolution for the neutrino's origin. This neutrino angular resolution is comparable to the angular resolution of the Fermi Large Area Telescope (LAT) for $\sim 1 \mathrm{GeV}$ photons. Utilising a deep LAT exposure and taking advantage of these angular resolutions, we have searched for faint and flaring $\mathrm{GeV} \gamma$-ray sources spatially coincident with IceCube's track-like events. No faint $\gamma$-ray sources or $\gamma$-ray flares were found to be spatially or temporally coincident with the neutrino candidates considered. The deep exposure did however reveal several new $\gamma$-ray point sources which were found to be spatially coincident with Active Galactic Nuclei. The non-detection of the $\gamma$-ray counterparts of the neutrino candidates is briefly discussed.
\end{abstract}

The 34th International Cosmic Ray Conference,

30 July- 6 August, 2015

The Hague, The Netherlands

\footnotetext{
* Speaker.
} 


\section{Introduction}

Neutrinos afford us a unique view of the Universe. Due to their neutral charge and small interaction cross-section, neutrinos they can escape, undeflected, from some of the most dense regions of the Universe. Furthermore, given their small interaction cross-section, neutrinos are able to travel cosmological distances to reach us here on Earth. With one cubic kilometre of Antarctic ice instrumented, the IceCube Neutrino Observatory is currently the largest neutrino detector in the world [1]. Recently, the IceCube collaboration reported evidence for the existence of extraterrestrial high-energy neutrinos [2]. This evidence was obtained through a 'HESE' analysis of the first two years of data taken with the 79 and 86 string IceCube detector. The addition of a third year of data resulted in this observational evidence evolving to a $5 \sigma$ discovery of these extraterrestrial neutrinos[3].

IceCube classifies the events observed by the pattern of the Cherenkov light seen in the detector array. Track events are those where the presence of a high-energy muon has produced a distinctive visible track of light. Shower events are those where the Cherenkov light from the particle shower initiated when the neutrino interacts is observed only in aggregate. For equal neutrino fluxes of all flavours, events with tracks make up only $20 \%$ of interactions [3]. Eight of the 37 HESE neutrino candidate events were classified as track events, 28 as shower events and one event was formed by two coincident cosmic-ray muon events. Importantly however, due to the 'lever arm' of the muon track, the angular resolution for events containing visible muon tracks is much better $\left(\leq 1^{\circ}\right)$ than for those that do not $\left(\sim 15^{\circ}\right)[3]$.

Described in detail by [4], the LAT detector onboard the Fermi satellite is a pair-conversion telescope, sensitive to a photon energy range of $\sim 20 \mathrm{MeV}$ to $>300 \mathrm{GeV}$. With a large field of view, $\simeq 2.4 \mathrm{sr}$, improved angular resolution, $68 \%$ containment angle of $\sim 0.8^{\circ}$ at $1 \mathrm{GeV}$, and large effective area, $\sim 8000 \mathrm{~cm}^{2}$ on axis for $10 \mathrm{GeV}$ photons, Fermi-LAT provides an order of magnitude improvement in performance compared to its EGRET predecessor. Since 2008 August 4, the vast majority of data taken by Fermi has been recorded in all-sky-survey mode, whereby the Fermi-LAT detector points away from the Earth and rocks north and south of its orbital plane on subsequent orbits. This rocking motion, coupled with Fermi-LAT's large effective area, allows Fermi to scan the entire $\gamma$-ray sky every two orbits, or approximately every three hours. This observational characteristic affords us, for the first time, quasi-continuous monitoring of the highenergy $\gamma$-ray sky and thus has important implications for the study of transient events. In addition to this, coupling Fermi-LAT's continual scanning of the sky with a long mission lifetime allows us to construct a deep exposure of the $\gamma$-ray sky, which is important for the study of faint $\gamma$-ray sources.

Neutrino emission from high-energy astrophysical sources capable of accelerating protons to relativistic energies has long been predicted. The neutrinos arise from the decay of charged mesons created in the interactions of the relativistic protons with either ambient gas (p-p) or ambient radiation $(\mathrm{p}-\gamma)$. If the $20 \mathrm{TeV}-2 \mathrm{PeV}$ extraterrestrial neutrinos detected by IceCube were indeed created this way, then their existence implies the existence of astrophysical objects capable of accelerating protons to energies of at least $O(10)$ to $O(100) \mathrm{PeV}$. The decay of neutral mesons created in these interactions produces $\gamma$-rays with energies comparable to the companion neutrinos. As such, the $\sim 30-250 \mathrm{TeV}$ extraterrestrial neutrino candidates detected by IceCube will be accom- 
panied by $\sim 10-100 \mathrm{~s} \mathrm{TeV} \gamma$-rays. These $\mathrm{TeV} \gamma$-rays are believed to cascade quickly down to $E_{\gamma}<100 \mathrm{GeV}$ energies due to the photon opacity of the emission region[5].

Assuming that the opacity of the neutrino $/ \gamma$-ray emission region is large enough to facilitate the transformation of the initial $\mathrm{TeV}$ photons down to $\mathrm{GeV}$ energies while not being so large as to absorb the $\gamma$-ray flux entirely, a search for the neutrino sources can be made with the Fermi-LAT detector. This possibility is an attractive one for the following reasons:

- the angular resolution of IceCube's track events is comparable with that of the Fermi-LAT detector for $E_{\gamma}>1 \mathrm{GeV}$ photons, which facilitates associating the neutrino events with FermiLAT detected $E_{\gamma} \sim 1 \mathrm{GeV} \gamma$-ray sources.

- the Fermi-LAT's continual monitoring of the high-energy $\gamma$-ray sky allows us to investigate whether the neutrino emission is associated with a transient $\gamma$-ray source.

- the long mission live time of Fermi, coupled with the LAT's large effective area, affords us the deepest extra-galactic survey of $1-300 \mathrm{GeV} \gamma$-ray photons.

In this work, we use the deep exposure of the first 70 months of Fermi-LAT operation to search for GeV counterparts to the track events of IceCube's 3-year HESE analysis. All neutrino candidates with a track-like topology are investigated, with the exception of event \#28, which displays evidence of a cosmic ray induced air shower in the IceTop detector accompanying the neutrino candidate, thus suggesting it is part of the atmospheric background. A complete likelihood analysis with the FERMI SCIENCE TOOLS was conducted for each neutrino candidate, with each candidate being considered independently. While sensitive to catalogue sources, conducting these studies also allows us to search for faint $\mathrm{GeV} \gamma$-ray sources not present in the existing Fermi catalogues and, in the absence of evidence for a source, determine upper limits to the associated $\mathrm{GeV} \gamma$-ray flux.

\section{Data analysis}

The data used in this study comprise all Fermi-LAT event and spacecraft data taken during the first 70 months of Fermi-LAT operation, from 2008 August 4 to 2014 June 4, which equates to a Mission Elapsed Time (MET) interval of 239557417 to 423589417 . All souRCE $\gamma$-ray events, in the $1<E_{\gamma}<300 \mathrm{GeV}$ energy range, within a $5^{\circ}$ radius of interest (RoI) centered on the right ascension and declination of the origin of HESE track-like events were considered ${ }^{1}$. Throughout the analysis, version $\mathrm{V} 9 \mathrm{R} 33 \mathrm{P} 0$ of the Fermi Science Tools was used in conjunction with the P7REP_SOURCE_V15 instrument response functions (IRFs). In accordance with the PASS7_REP criteria $^{2}$, a zenith cut of $100^{\circ}$ was applied to the data to remove any cosmic ray induced $\gamma$-rays from the Earth's atmosphere. The good time intervals were generated by applying a filter expression of "(DATA_QUAL $==1) \& \&($ LAT_CONFIG $==1) \& \&$ ABS $($ ROCK_ANGLE $)<52$ " to the data. A summary of the analysis criteria is given in Table 1.

\footnotetext{
${ }^{1}$ The right ascension and declination for IceCube's HESE track events were taken from Table 1 of [3].

${ }^{2}$ We note that recently the Fermi-LAT collaboration has released PASS 8 LAT data, which provide a much improved angular and energy resolution for LAT events. An updated analysis using PASS 8 data is currently being undertaken.
} 


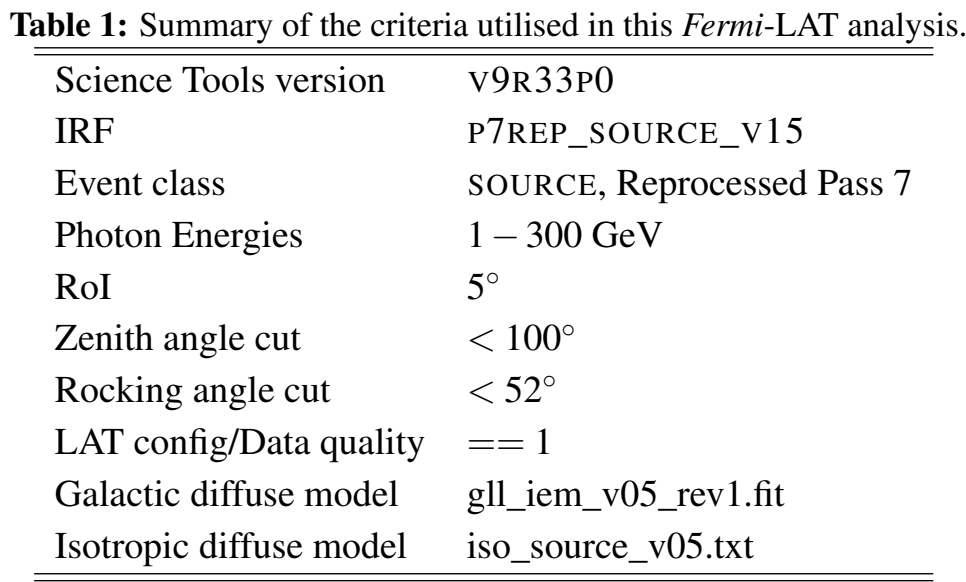

During the likelihood analysis, a model file consisting of both point and diffuse sources of $\gamma$-rays was employed. Care must be taken to ensure the accuracy of the model used since, if new sources are present in the data, and not properly accounted for within the model file utilised during the likelihood analysis, the significance of the $\gamma$-ray flux from the neutrino candidates can be artifically increased. The process through which we confirmed the accuracy of our models is described in detail in [6]. However, generally speaking, within this study the diffuse source models used in the global models for each neutrino candidate are given in Table 1. In addition to these diffuse models, all point sources within $10^{\circ}$ of the candidate neutrino position $\left(\alpha_{J 2000}, \delta_{J 2000}\right)$ were considered in the likelihood model. The position and spectral shape for these point sources were taken from the second Fermi catalogue.

An initial BINNED likelihood analysis was conducted to confirm the accuracy of the 'diffuse + 2FGL point source' model. The best-fit model from this initial likelihood fit was used, in conjunction with the Fermi tool GTMODEL, to construct a 'model map' of a $7^{\circ} \times 7^{\circ}$ region centered on the $\left(\alpha_{J 2000}, \delta_{J 2000}\right)$ of each candidate neutrino track event. This model map was then compared with the 70 -month sky map of the $1-300 \mathrm{GeV} \gamma$-rays to create a residuals count map ${ }^{3}$. Any positive excess of events in the residuals map indicates the presence of a new source of $\gamma$-rays.

For the majority of the candidate neutrino events, the residuals maps do not reveal any obvious excess in $\gamma$-ray emission coincident with the neutrino's point of origin, the two exceptions being neutrino candidates \#5 and \#23. The $7^{\circ} \times 7^{\circ}$ sky map, model map and residuals maps for these two events can be seen in Figures 1 and 2 respectively. Interestingly, as can be seen in Figure 1, there is also a known, faint $\gamma$-ray source in the median error radius for the neutrino candidate \#5. We will return to this in Section 3.

To search for faint $\gamma$-ray sources associated with the candidate neutrinos' origin that may not be apparent in the residual sky map, a further BINNED analysis was conducted, with an additional point source included in the model file at the position of the candidate neutrino event. The spectral form of these additional point sources was described by a power law spectrum, $d N / d E=\mathrm{A} \times\left(E / E_{o}\right)^{-\Gamma}$, with both $\mathrm{A}$ and $\Gamma$ left free to vary. The $\left(\alpha_{J 2000}, \delta_{J 2000}\right)$ for each 'neutrino' point source were fixed to the values published by the IceCube Collaboration. All 'neutrino' point sources added to the

\footnotetext{
${ }^{3}$ The residuals count map was constructed via (sky map-model map)/model map (e.g. see [7])
} 

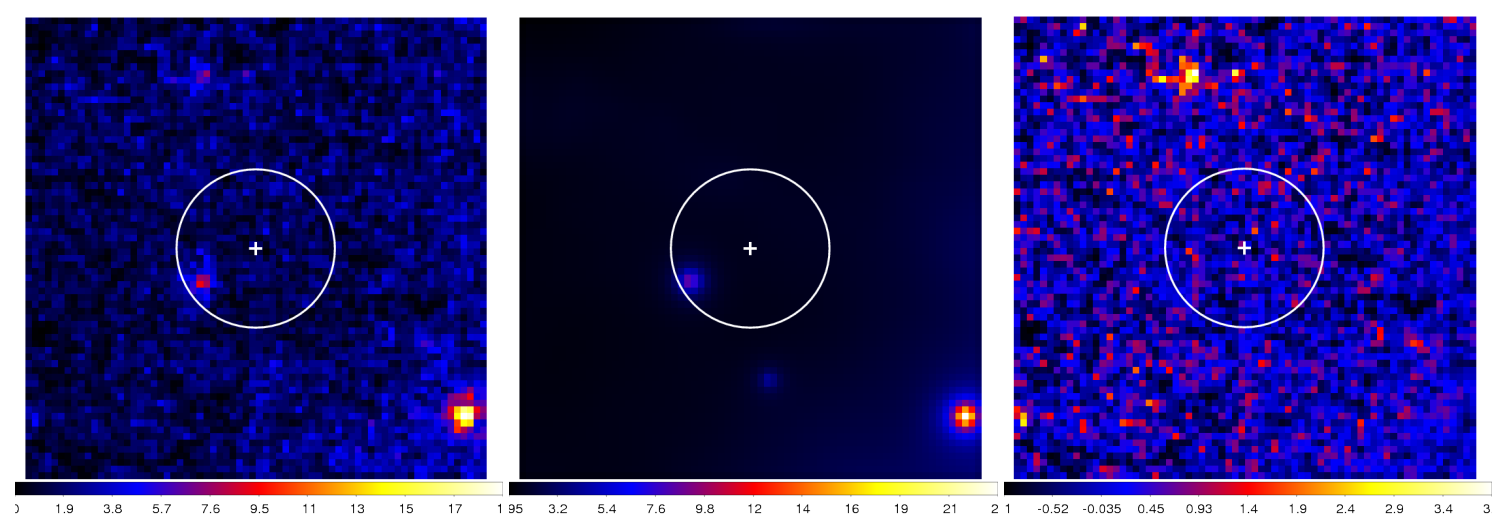

Figure 1: Event 5: Left: $7^{\circ} \times 7^{\circ}$ sky map of the $1-300 \mathrm{GeV}$ flux for the entire 5.8 year data set. Middle: $7^{\circ} \times 7^{\circ}$ model map produced with the Fermi tool GTMODEL, utilising the best-fit model from the 5.8 year binned likelihood analysis. Right: $7^{\circ} \times 7^{\circ}$ residuals map of the $1-300 \mathrm{GeV}$ events. The residuals map is produced by (sky map-model map)/model map). All maps have been smoothed with a $1^{\circ}$ Gaussian. The cross at the centre of each map indicates the $\left(\alpha_{J 2000}, \delta_{J 2000}\right)$ of the neutrino candidate, while the circle present in each map indicates the median error radius for the neutrino candidate as shown in Table 2 . The colour scales for the sky and model maps are in units of $\gamma$-ray counts, while the residuals maps are in units of percentage. There is an excess in the residuals map consistent with a point source at $\left(\alpha_{J 2000}, \delta_{J 2000}\right)=$ $(111.419,2.221)$.
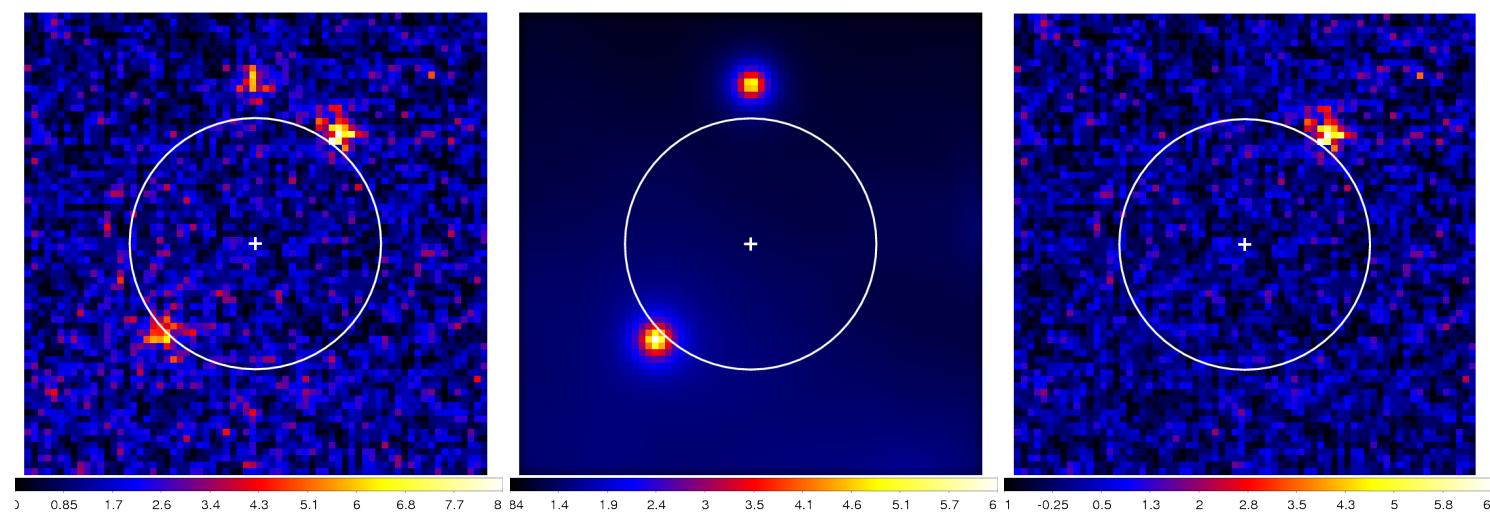

Figure 2: $7^{\circ} \times 7^{\circ} \mathrm{sky}$, model and residuals maps for Event 23. There is a excess in the residuals map consistent with a point source at $\left(\alpha_{J 2000}, \delta_{J 2000}\right)=(207.409,-11.553)$.

model file for this additional 70-month BINNED analysis had a test statistic ${ }^{4}$ of 0 . As such, no evidence for $1-300 \mathrm{GeV} \gamma$-ray emission spatially coincident with the $\left(\alpha_{J 2000}, \delta_{J 2000}\right)$ of the HESE track events was found. Upper limits, at the $95 \%$ confidence level, were therefore calculated for the $\gamma$-ray emission, assuming a point source for the candidate neutrino events. These upper limits are summarised in Table 2.

\footnotetext{
${ }^{4}$ The test statistic, TS, is defined as twice the difference between the log-likelihood of two different models, $T S=$ $2\left[\log L-\log L_{0}\right]$, where $L$ and $L_{0}$ are defined as the likelihoods when the source is included or not respectively ([8]).
} 
Table 2: Summary of upper limits for the $1-300 \mathrm{GeV} \gamma$-ray flux spatially coincident with the $\left(\alpha_{J 2000}\right.$, $\left.\delta_{J 2000}\right)$ of the HESE track events.

\begin{tabular}{ccccccc}
\hline \hline $\begin{array}{c}\text { IceCube } \\
\text { event }\end{array}$ & $\begin{array}{c}\text { Deposited } \\
\text { Energy }(\mathrm{TeV})\end{array}$ & $\begin{array}{c}\alpha_{J 2000} \\
(\mathrm{deg})\end{array}$ & $\begin{array}{c}\delta_{J 2000} \\
(\mathrm{deg})\end{array}$ & $\begin{array}{c}\text { Angular error } \\
(\mathrm{deg})\end{array}$ & $\begin{array}{c}\text { Hemisphere } \\
\text { of origin }\end{array}$ & $\begin{array}{c}\gamma \text {-ray flux upper limit } \\
\left(\mathrm{ph} \mathrm{cm}^{-2} \mathrm{~s}^{-1}\right)\end{array}$ \\
\hline$\# 3$ & $78.7_{-8.7}^{+10.8}$ & 127.9 & -31.2 & $\leq 1.4$ & South & $1.89 \times 10^{-10}$ \\
$\# 5$ & $71.4_{-9.0}^{+9.0}$ & 110.6 & -0.4 & $\leq 1.2$ & South & $9.09 \times 10^{-11}$ \\
$\# 8$ & $32.6_{-11.3}^{+10.3}$ & 182.4 & -21.2 & $\leq 1.3$ & South & $8.64 \times 10^{-11}$ \\
$\# 13$ & $253_{-22}^{+26}$ & 67.9 & 40.3 & $\leq 1.2$ & North & $1.99 \times 10^{-10}$ \\
$\# 18$ & $31.5_{-3.3}^{+4.6}$ & 345.6 & -24.8 & $\leq 1.3$ & South & $1.05 \times 10^{-10}$ \\
$\# 23$ & $82.2_{-8.4}^{+8.6}$ & 208.7 & -13.2 & $\leq 1.9$ & South & $1.32 \times 10^{-10}$ \\
$\# 37$ & $30.8_{-3.5}^{+3.3}$ & 167.3 & 20.7 & $\leq 1.2$ & North & $5.18 \times 10^{-11}$ \\
\hline \hline
\end{tabular}

\section{IceCube event \#5}

In addition to a new $\gamma$-ray source within the RoI, the neutrino candidate \#5 has a known $\gamma$-ray bright AGN within the $1 \sigma$ uncertainty of the event's position of origin. Located $(1.04 \pm 0.04)^{\circ}$ from neutrino \#5 is PKS 0723-008. Present in both the 1 year and 2 year Fermi-LAT AGN catalogues, PKS 0723-008 is a BL Lac object with a redshift of 0.128. In our 70-month BINNED analysis, PKS 0723-008 is detected with a TS of 176.8, a spectral index $\Gamma=2.07 \pm 0.12$ and a flux of $(9.35 \pm 1.20) \times 10^{-10} \mathrm{ph} \mathrm{cm}^{-2} \mathrm{~s}^{-1}$. Both PKS 0723-008's 70-month flux and spectral index are consistent with the values published in the 2LAC catalogue.

To quantify the significance of the spatial coincidence of $2 \mathrm{LAC}$ AGN with a single track-like neutrino event, the positions of all $2 \mathrm{LAC}$ sources were scrambled in $\alpha_{J 2000}$ and $\delta_{J 2000}$, and the new positions were cross-correlated with the $\alpha_{J 2000}$ and $\delta_{J 2000}$ of all neutrino candidates with $E_{v} \geq 60$ $\mathrm{TeV}^{5}$ considered in this study. Repeated 10,000 times, this approach revealed that the chance probability of having a 2LAC AGN positionally coincident with one of the $E_{v} \geq 60 \mathrm{TeV}$ tracklike neutrino candidates to be $\sim 37 \%$. This large probability suggests that the spatial coincidence between event \#5 and PKS 0723-008 is a chance event.

Nonetheless, to investigate the temporal characteristics of PKS 0723-008's $1-300 \mathrm{GeV} \gamma$-ray flux around the time the \#5 neutrino candidate was detected, the data were binned into 50 day periods, with an UNBINNED GTLIKE routine applied to each bin separately. Only temporal bins with test statistic $T S>10$ were considered. The resultant lightcurve is shown in Figure 3. While the lightcurve does reveal periods of enhanced $\gamma$-ray flux from PKS 0723-008 when compared to the 70-month average, there is no obvious flare event associated with the detection of the candidate neutrino event. Indeed, during the 50-day period in which the neutrino candidate was detected by IceCube, no significant $\gamma$-ray flux was observed.

\footnotetext{
${ }^{5}$ As discussed in [9], the background neutrino events considered in this study are expected to concentrate at lower energies, $E_{V}<60 \mathrm{TeV}$.
} 


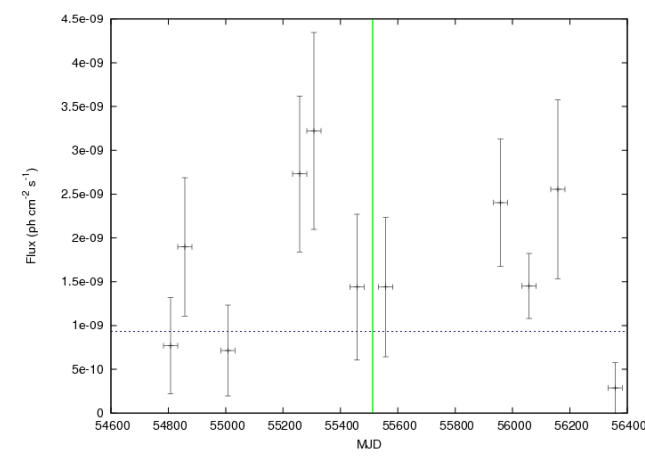

Figure 3: The lightcurve of the $1-300 \mathrm{GeV}$ flux from PKS 0723-008 binned in 50 day periods. The horizontal dashed line shows the flux from the 70-month BINNED analysis, while the vertical solid line shows the time the \#5 neutrino candidate was detected.

\section{Discussion}

Neutrino candidate \#5 is the only event found to have a $\gamma$-ray point source within the median error of its origin. However, Monte Carlo simulations revealed the probability that a 2LAC source is positionally coincident with a HESE neutrino candidate to be $\sim 37 \%$, suggesting that this positional coincidence is merely a chance event. As such, this study has found no $\gamma$-ray emission positionally coincident with the track-like events of IceCube's HESE analysis. Possible explanations for these non-detections are as follows:

(i) The diffuse neutrino flux observed by IceCube is the aggregate of a very large number of faint neutrino sources which, correspondingly, are faint $\gamma$-ray sources. IceCube's own point source limits mean that a diffuse astrophysical flux at the level of $10^{-8} \mathrm{GeV} \mathrm{cm}^{-2} \mathrm{~s}^{-1} \mathrm{sr}^{-1}$, as reported in ([10]), must be distributed over at least $O(50)$ sources. In order to be able to relate the neutrino flux from an individual source to a corresponding photon flux at $\mathrm{GeV}$ energies requires detailed modelling which is beyond the scope of this paper. However we note that typical models (eg. [11]) relating a source's photon and neutrino bolometric luminosities, would suggest that a photon flux, at the level of the upper limits presented in Table 2, is associated with an $E^{-2}$ neutrino flux at least an order of magnitude below the current IceCube point source limits. Thus, if the TeV photons associated with the neutral mesons are able to quickly cascade down to the $\mathrm{GeV}$ energies considered in this dedicated analysis of Fermi-LAT observations AND if the typical relationships between the neutrino and photon bolometric luminosities hold, then our photon limits suggest that the diffuse neutrino flux observed by IceCube is drawn from the aggregate of at least $O(500)$ sources.

(ii) In the context of the emission region's $\gamma$-ray opacity, the $\gamma$-ray non-detection of the neutrino sources of our study can be interpreted as being due to the opacity being either too small or too large. If the opacity is too low, the $\mathrm{TeV} \gamma$-rays are able to freely escape and thus the $\gamma$-ray flux is not able to cascade down to the photon energy range considered in this study. Likewise, if the opacity is too high, the $\mathrm{TeV} \gamma$-ray flux would quickly be processed to energies well below the photon energy range considered in this study. Observations with the future Cherenkov Telescope Array [12] would shed light onto the former possibility, while detailed multi-wavelength studies would be needed to investigate the latter possibility.

(iii) Finally, we note that IceCube's HESE analysis claimed the discovery of extraterrestrial 
neutrinos at a $\sim 5.6 \sigma$ confidence level, with 37 candidate neutrino events detected relative to an expected background of $8.4 \pm 4.2$ cosmic ray muon events and $6.6_{-1.6}^{+5.9}$ atmospheric neutrinos. As such, anywhere between a quarter and two-thirds of the neutrino candidates detected are expected to be background events from cosmic rays interacting with the Earth's atmosphere.

\section{Conclusion}

The angular resolution of a $E_{\gamma} \approx 1 \mathrm{GeV}$ photon detected by the Fermi-LAT detector is comparable to the median angular error of IceCube's HESE neutrino candidates detected as track-type events. As such, associating the track-like events of IceCube's HESE analysis with known FermiLAT detected $E_{\gamma}>1 \mathrm{GeV}$ sources is simplified greatly when compared to trying to find counterparts to IceCube's shower-like events. Motivated by this, we analysed 70 months of Fermi-LAT observations to search for $1-300 \mathrm{GeV}$ emission spatially coincident with the origin of IceCube's HESE track events. With the exception of event \#5, no $E_{\gamma}>1 \mathrm{GeV}$ emission spatially coincident with the origin of the neutrino events was found. For these events, $95 \%$ confidence level upper limits were calculated.

Event \#5 is the only HESE track-like event that has a $\gamma$-ray point source within the median error of its origin, with PKS 0723-008 being separated from the origin by $\sim 1^{\circ}$. A Monte Carlo study found that the probability of such a spatial coincidence occuring by chance is relatively high, with it occuring in $37 \%$ of the cases we simulated. As such, an insignificant spatial coincidence, combined with the observed $\gamma$-ray properties, suggests that PKS 0723-008 is not the source of the \#5 neutrino candidate. We note that the Fermi-LAT collaboration has recently released the PASS 8 LAT data, which provide a much improved angular and energy resolution for LAT events. An updated analysis using PASS8 data is currently being undertaken.

\section{References}

[1] IceCube Collaboration, https://icecube.wisc.edu/

[2] IceCube Collaboration, 2013, Science, 342, 1

[3] Aarsten, M.G., et al. 2014a, PhRvL, 113, 101101

[4] Atwood, W.B., et al. 2009, ApJ, 697, 1071

[5] Mannheim, K., 1993, A\&A, 269, 67

[6] Brown, A.M, Adams, J. \& Chadwick, P.M., 2015, MNRAS, 451, 4842

[7] Brown, A.M., Chadwick, P.M. \& Landt, H., 2014, MNRAS, 4345, 50

[8] Mattox J.R., et al. 1996, ApJ, 461, 396

[9] Padovani, P. \& Resconi, E., 2014, MNRAS, 443, 474

[10] Aarsten, M.G., et al. 2014b, ApJ, 769, 109

[11] Halzen, F. \& Zas, E., 1997, ApJ, 488, 66

[12] Acharya, B.S., et al. 2013, APh, 43, 3 